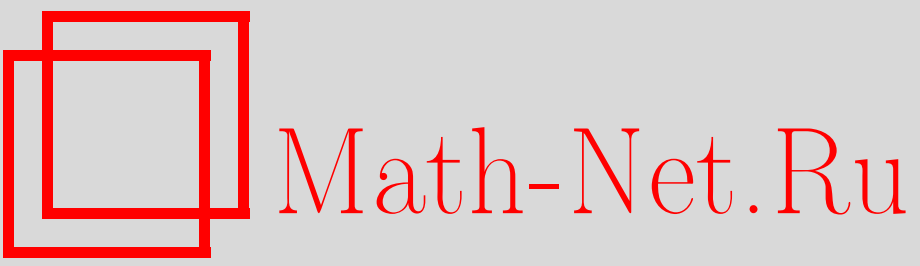

О. Н. Попов, Об одной конструкции модулей над кольцом многочленов, УМН, 2001, том 56, выпуск 6, 163-164

DOI: https://doi.org/10.4213/rm467

Использование Общероссийского математического портала Math-Net.Ru подразумевает, что вы прочитали и согласны с пользовательским соглашением

http://www.mathnet.ru/rus/agreement

Параметры загрузки:

IP : 52.90 .164 .192

26 апреля 2023 г., 13:08:15 


\title{
ОБ ОДНОЙ КОНСТРУКцИИ МОДУЛЕЙ НАД КОЛЬЦОМ МНОГОЧЛЕНОВ
}

\author{
О. Н. Попов
}

В статье [1] при исследовании кватернионно-дифференцируемых функций многих переменных возник следующий модуль над кольцом многочленов: $\mathscr{M}_{n}=R^{4} /\left\langle A_{n}\right\rangle$, где $A_{n}$ - матрица $U_{1}|\ldots| U_{n},\left\langle A_{n}\right\rangle$ - подмодуль, порожденный ее столбцами,

$$
U_{i}=\left(\begin{array}{cccc}
x_{i} & y_{i} & z_{i} & t_{i} \\
-y_{i} & x_{i} & t_{i} & -z_{i} \\
-z_{i} & -t_{i} & x_{i} & y_{i} \\
-t_{i} & z_{i} & -y_{i} & x_{i}
\end{array}\right),
$$

а $R=\mathbb{k}\left[\left\{x_{i}, y_{i}, z_{i}, t_{i}\right\}_{i=1}^{n}\right], \mathbb{k}-$ поле.

Авторы показывали, что проективная размерность этого модуля равна $2 n-1$, и вьводили отсюда некоторые утверждения о пучке кватернионно-дифференцируемых функций $n$ переменных. Авторы вычисляли проективную размерность по формуле Ауслендера-Буксбаума, посредством явного (при помощи базисов Грёбнера) предъявления $\mathscr{M}_{n}$-последовательности, а не построением резольвенты, так как этот путь они считали слишком сложным. В статье также была найдена размерность Крулля $\mathscr{M}_{n}$, для чего рассматривалось касательное пространство к носителю этого модуля в $\mathbb{C}^{4 n}=\operatorname{Specm~} R$ для $\mathbb{k}=\mathbb{C}$. Таким образом была доказана коэн-маколеевость этого модуля, т.е. равенство размерности Крулля и глубины.

В статье [2] авторы продолжили исследования этого модуля с помощью базисов Грёбнера, найдя (градуированные) числа Бетти, ряд Гилберта и кратность $\mathscr{M}_{n}$.

В [1] матрица $A_{n}$ получилась из системы линейных дифференциалных уравнений в частных производных, задающей кватернионно-дифференцируемые функции, транспонированием и заменой операторов частных производных по пер еменным на сами переменные. Однако можно заметить (cp. [2; введение]), что матрица $U_{i}$ есть матрица левого умножения на $x_{i}-y_{i} i-z_{i} j-t_{i} k$ в базисе $1, i, j, k$. Такой взгляд на матрицу $A_{n}$ позволил, как заметил Е. С. Голод, полностью понять структуру модуля $\mathscr{M}_{n}$, в частности, его проективной резольвенты. Комплексифицируем алгебру кватернионов. Так как при замене базиса в алгебре матрица $U_{i}$ заменяется на сопряженную и в ней происходит линейная замена переменных, то структура модуля $\mathscr{M}_{n}$ от этого не изменится. Поэтому изоморфизм $\mathbb{H} \otimes_{\mathbb{R}} \mathbb{C} \cong M_{2}(\mathbb{C})$ - матричной алгебре - позволяет в базисе из матричных единиц придать матрице $U_{i}$ вид

$$
\left(\begin{array}{cccc}
a_{i} & b_{i} & & 0 \\
c_{i} & d_{i} & \multicolumn{1}{c}{} \\
0 & a_{i} & b_{i} \\
& & c_{i} & d_{i}
\end{array}\right)
$$

т.е. $\mathscr{M}_{n}=\mathscr{M}_{n}^{\prime} \oplus \mathscr{M}_{n}^{\prime}$, где $\mathscr{M}_{n}^{\prime}$ - фактор $R^{2}$ по столбцам общей $2 \times 2 n$-матрицы. А про $\mathscr{M}_{n}^{\prime}$ известно [3], что это - коэн-маколеев (CM) модуль проективной размерности $2 n-2+1=2 n-1$ и что его минимальная резольвента - это комплекс Игона-Норкотта $\left([3 ; 2 . C], \mathscr{D}_{1}(g)\right)$. Это описание резольвенты позволяет упростить доказательства основных резултатов статьи [2].

Отсюда возникло следующее обобщение этой задачи. Пусть $A$ - конечномерная ассоциативная алгебра с 1 над полем $\mathbb{k}$ с базисом $e_{1}, \ldots, e_{d}$, а $\rho: A \rightarrow M_{n}(\mathbb{k})$ - ее матричное представление, отвечающее $A$-модулю $M, \operatorname{dim}_{\mathbb{k}} M=n$. Зададим натуралшное $l$ и рассмотрим кольцо многочленов $R=\mathbb{k}\left[x_{11}, \ldots, x_{d l}\right]$ и модуль $F_{l}(M)$ над ним, являющийся фактормодулем свободного $R$-модуля $R^{n}$ по подмодулю, порожденному столбцами матриц $\operatorname{Id}_{j}=\sum_{i} \rho\left(e_{i}\right) x_{i j}, j=1, \ldots, l$. Мы исследуем вопрос о коэн-маколеевости и размерности модулей $F_{l}(M)$ и их аннуляторов. Автором получена

Работа выполнена при частичной поддержке Российского фонда фундаментальных исследований (грант № 99-01-01144). 
Теорема 1. Пусть либо $l=1$, либо $A=\bigoplus_{i} S_{i} \otimes_{Z\left(S_{i}\right)} K_{i}$, где $S_{i}-$ простые алгебрь над $\mathbb{k}, Z\left(S_{i}\right)$ - их центры, а $K_{i}$ - конечномернье коммутативные алгебры над соответствующими $Z\left(S_{i}\right), u \operatorname{dim}_{Z\left(S_{i}\right)} S_{i}=n_{i}^{2}$. Тогда

1) $F_{l}(\cdot)$ есть точный вполне строгий функтор из категории конечномерных $A$-модулей в категорию градуированных $R$-модулей и однородных гомоморфизмов степени 0;

2) если $l=1$ или $n_{i}=n$ для всех $i$, то функтор $F_{l}(\cdot)$ переводит конечномерные $A$-модули в $C M R$-модули проективной размерности $(l-1) n+1$ (что равно 1 npu $l=1)$;

3) при $l=1$ для любого $M$ аннулятор $F_{l}(M)$ - главный идеал.

По ходу доказательства получается некоторая информация о минимальной резольвенте модулей $F_{l}(M)$. А именно, для $l>1$ теорему достаточно доказать для одного слагаемого и над алгебраически замькнутым полем $\mathbb{k}$, т.е. для алгебры $M_{n}(K)$, где $K$ - коммутативна. В этих условиях минимальную резольвенту модуля $F_{l}(M)$ можно строить функториально по $M$, обобщая замечание Е. С. Голода: при $l=1$ она есть

$$
0 \longrightarrow R^{n} \stackrel{\mathrm{Id}_{1}}{\longrightarrow} R^{n} \longrightarrow F_{l}(M) \longrightarrow 0,
$$

а при $l>1$ матрицу $\left(\operatorname{Id}_{1}|\ldots| \operatorname{Id}_{l}\right)$ можно представить как блочную $(n \times \ln )$-матрицу, блоки которой есть аналогичные Id-матрицы для соответствующего $K$-модуля $\mathscr{M}$. Так как эти блоки коммутируют между собой, то их можно использовать как элементы матрицы для построения комплекса Игона-Норкотта $R$-модуля $\mathscr{M} \otimes_{\mathbb{k}} R$, и этот комплекс будет минимальной резольвентой. Это позволяет найти различные численные инварианты модулей $F_{l}(M)$, обобщая таким образом основные результаты из [2].

Следующее частичное обращение теоремы 1 демонстрирует естественность ограничений, накладьваемых на алгебру при $l>1$ :

Теорема 2. Пусть поле $\mathbb{k}$ совериенно и алгебра А такова, что для некоторого $l>1$ либо (i) функтор $F_{l}(\cdot)$ точен, либо (ii) для любого конечномерного $A$-модуля $M$ модуль $F_{l}(M)$ коэн-маколеев. Тогда $A$ является прямой суммой указанного в условии теоремы 1 вида, и в случае (ii) $n_{i}$ не зависят от $i$.

Было бы интересно получить из комплекса Игона-Норкотта, являющегося минимальной резольвентой модуля $\mathscr{M}_{n}$ из [1], явную ацикличную резольвенту для пучка кватернионно-дифференцируемых функций и с еепомощью дать для результатов из [1] аналитические доказательства, которых не хватает авторам.

Автор хотел бы поблагодарить Е. С. Голода за постоянное внимание и поучительные замечания и Е. А. Котельникову, подтолкнувшую его к работе, а также рецензента за упрощение некоторых рассуждений.

\section{СПИСОК ЛИТЕРАТУРЫ}

[1] W. W. Adams, P. Loustaunau, V.P. Palamodov, D. C. Struppa // Ann. Inst. Fourier. 1997. V. 47. № 2. P. 623-640. [2] W. W. Adams, P. Loustaunau // Pacific J. Math. 2000. V. 196. №1. P. 1-15. [3] W. Bruns, U. Vetter. Determinantal Rings. Berlin: Springer-Verlag, 1988. (Lecture Notes in Math. V. 1327.) 